\title{
HETITA E INDOEUROPEO
}

The linguistic characteristics of Hittite show remarkable differences in relation to the traditional reconstruction of Indoeuropaean. Even when certain linguists try to interpret these discrepancies as innovations of Hittite, there is a powerful

current of opinion that detects many archaisms in the Anatolian branch.

E1 desciframiento y la identificación como lengua indoeuropea del hetita por parte de Hrozny, si bien resultó sorprendente en su momento, no tardó en imponerse, de modo que desde muy pronto pasó por doctrina averiguada y nadie ha puesto más en tela de juicio la indoeuropeidad de la rama anatolia.

La integración del hetita dentro de los esquemas del indoeuropeo, reconstruido antes de que los indogermanistas conociesen los datos que nos proporcionan las lenguas anatolias, ha sufrido en cambio una suerte muy diversa, encontrándonos hoy muy lejos de alcanzar una unanimidad de criterio.

Para expresarlo en breves palabras, el problema es el siguiente: el hetita llega a conocimiento de los lingüistas después de la época de codificación de la ciencia de la indogermanística, que se realizó por consiguiente sin el concurso de los datos proporcionados por la rama anatolia. El hetita, a pesar de datar de la más alta antigüedad - es la lengua indoeuropea de testimonio más antiguo- presenta una situación fonética y morfológica muy distinta de los esquemas tradicionales. $Y$ ello resulta tanto más llamativo, cuanto que las lenguas indoeuropeas se parecen más entre sí cuanto más antiguas son, mientras que ese parecido se va desdibujando con el correr de los siglos. Sin embargo, el hetita, a pesar de su antigüedad, ofrece un panorama muy diferente al de las otras lenguas indoeuropeas antiguas, como pueden ser el griego o el indoiranio. 
El enfrentamiento de los datos del hetita con los resultados de la indogermanística clásica resultó molesto para la validez misma del método comparativo, y desde pronto se inició por parte de los indoeuropeístas una ardua labor de interpretación, tendente a devolver la tranquilidad, reafirmando la validez del método tradicional y de sus resultados concretos.

Una línea de pensamiento, matizada de diferentes formas por distintos indoeuropeístas, consiste en suponer una separación temprana de la rama anatolia, con lo que las posibles discrepancias con el indoeuropeo de la reconstrucción clásica pueden salvarse alegando simplemente que el hetita se apartó del resto del indoeuropeo en un estadio lingüístico anterior al reconstruido normalmente. Este esquema de pensamiento suele asociarse al nombre de Sturtevant, que sin embargo no es el único ni el primero en proponerlo.

El primero que apunta la posibilidad de una temprana separación del hetita - hasta donde alcanza mi información- es Forrer ${ }^{1}$. Sus ideas fueron acogidas de una parte por Ungnand, y de otra por Sturtevant.

Ungnand $^{2}$ acuña el término Protindogermanisch para designar la totalidad de la familia con anterioridad a la separación del hetita, y reserva el nombre de Urindogermanisch para el indoeuropeo clásico, posterior a la separación de los anatolios. Para el autor, esa primera bifurcación dialectal sería anterior a la isoglosa centum/satam, por lo que no cabe decir en rigor ele el hetita sea una lengua centum, sino que refleja un estadio lingürstico previo a la propagación de la mencionada isoglosa.

Las ideas de Ungnand son aceptadas por Kretschmer ${ }^{3}$ y aplicadas a la interpretación de los estratos de población en la cuenca del Ėgeo: I) No-indoeuropeo, 2) Protoindoeuropeo, 3) Indoeuropeo.

Fue, sin embargo, por el camino de la obra de Sturtevant por donde llegarían a tener mayor difusión las ideas de Forrer, debido a que fue él quien acertó a darles una formulación general dentro de los esquemas de la doctrina del árbol genealógico, e igualmente quien emprendió la tarea de reunir pruebas de elementos arcaicos en hetita que demostraran su temprana separación. El conjunto del pensamiento de Sturtevant no aparece desde luego dibujado desde un primer momento, sino que va siendo elaborado a lo largo de una serie de trabajos relativamente numerosa.

1 Mitteilungen der Deutschen Orient-Gesellschaft 6r, p. 26 ss.

2 Z. Ass. $35=\mathrm{NF}$ I, p. 1 ss.

-Die protoindogermanische Schicht,, Glotta 14, 1925, pp. 300-319. 
En una de sus primeras publicaciones al respecto ${ }^{1}$ Sturtevant todavía vacila. Por una parte se adhiere a la sugerencia de Forrer de una temprana separación del hetita. Al antepasado común le llama Preindoeuropeo, término que más adelante, al ir perfilándose su pensamiento, habría de ser sustituido por el de Indo-Hetita, siguiendo una propuesta de Bloomfield, quien le hizo ver que el término Preindoeuropeo resultaba ambiguo al aplicarse por una parte a un estadio antiguo del indoeuropeo propiamente dicho y por otra al tronco común del indoeuropeo propiamente dicho y del hetita.

Por otra parte, sin embargo, no duda Sturtevant en considerar innovaciones del hetita todos aquellos hechos lingüísticos en que esta lengua se aparta del indoeuropeo clásico. Las excepciones a lo que acabamos de decir son pocas. La más importante es la que ya había citado Ungnand: situación previa a la propagación de la isoglosa centum/satam. Una situación tan generalmente innovadora del hetita no debía dejar demasiado convencido a Sturtevant cuando añadía que «resulta paradójico que la lengua indoeuropea más antigua conocida presente un estadio de desarrollo tan avanzado en vocabulario, morfología y fonética».

En el mismo año publica otro trabajo ${ }^{2}$ en el que aporta ya algunos arcaísmos nuevos para la defensa de la pronta separación del hetita:

I) Conservación de $h$. Su eliminación sería posterior a la separación del hetita.

2) Carácter arcaico del sistema de desinencias medio-pasivas del hetita. A partir de la situación del hetita pueden explicarse todos los tipos atestiguados en las otras lenguas indoeuropeas, y no viceversa.

3) Alternancia $m / w$ en sufijos y desinencias. Antes de la separación del hetita se habría producido una disimilación del grupo -ureen - $u m$ - de modo que el sufijo o desinencia se habria escindido en dos alomorfos fonéticamente condicionados. Tras la separación del hetita habría tenido lugar una alteración de esta situación primitiva: las dos variantes dejan de estar fonéticamente condicionadas y llegan a oponerse entre sí para expresar, por ejemplo, plural/dual. El hetita conserva en cambio el status primitivo en que el condicionamiento fonético y la situación alomórfica siguen vigentes.

1 Language 2, 1929, pp. 25-34.

- TAPhA 60, 1929, pp. 25-37. 
4) El elemento to- es en hetita un "sentence-conective", función a partir de la cual se explica bien la de demostrativo con tendencia a encabezar la frase con que aparece en otras lenguas indoeuropeas. También aquí el hetita conservaría la situación que antes de su separación habría sido general.

Los argumentos que aporta Sturtevant en este trabajo van a ser fundamentalmente los mismos que seguirá repitiendo en diversas publicaciones posteriores, con algunas modificaciones y añadidos.

Un año después ${ }^{1}$ aborda el viejo problema de las series guturales indoeuropeas. También a este respecto encuentra motivos para considerar arcaizante al hetita en relación con las demás lenguas de la familia. Por otra parte, su pensamiento va poco a poco concretándose: el hetita es un testimonio parelelo al indoeuropeo, necesarios uno y otro para reconstruir la situación lingüística anterior, el Indo-Hetita. Comparando, pues, por una parte los datos deducidos de las lenguas indoeuropeas, y por otra los del hetita, llega a conclusiones que podemos resumir como sigue:

a) En indo-hetita existiría una sola serie gutural, situación que, una vez más, conserva el hetita.

b) Estos fonemas guturales irían a veces seguidos en la cadena hablada por -w-, que tras consonante adquiriría un valor vocálico. También esa particularidad estaría conservada en hetita.

c) Una vez separado el hetita, en indoeuropeo se producen dos fenómenos:

I) Se da una tendencia a la eliminación de - $w$ - tras consonante, que sólo después de la desmembración del indoeuropeo llegará hasta sus últimas consecuencias en las lenguas satam. Andando el tiempo también se realiza por completo ese proceso en algunas lenguas centum.

2) Ante vocales delanteras se produce una articulación palatal que es fuente de la tercera serie indoeuropea $k, g, g h$.

Algunos años más tarde ${ }^{2}$ Sturtevant recoge uno de los argumentos expuestos en anteriores trabajos para desarrollarlo más a fondo: el

\footnotetext{
1 The gutturals in Hittite and Indo-Europeans, Language 6, 1930, pp. 213228.

-The pronoun so, to, tod and the indo-hittite hypothesiss, Langrage 15. 1939, Pp. II-I9.
} 
del demostrativo indoeuropeo y su origen en los unidores de frase indohetitas conservados como tales en hetita. En indo-hetita estos unidores habrian sido tres: *nu 'ahora', *to 'entonces' y *so 'y'. El indoeuropeo por su parte crea a partir de ahí la heteroclisis *so/to, añadiendo ulteriormente la forma de femenino, mientras que el hetita habria creado los conglomerados $n a-a \check{s}, t a-a \breve{s}$.

Con carácter póstumo apareció una exposición resumida del conjunto de la argumentación ${ }^{1}$. No merece la pena detenerse en su contenido ya que no es sino una repeticián ordenada de los argumentos enumerados hasta ahora. $\mathbf{Y}$ con ello podemos pasar ya a mencionar las dos obras principales de Sturtevant sobre el tema del hetita 2

En la primera de ellas se queja el autor de que diversos indogermanistas (Benveniste, Kuryłowicz, Sapir) se hubieran aplicado al estudio de las laringales hetitas sin tener en cuenta la hipótesis del indohetita, o habiéndola atacado abiertamente (Bonfante, Keith, Pedersen), viéndose en consecuencia en la necesidad de repetir una vez más la argumentación. Agrupa sus razones en tres tipos:

I) Las lenguas indoeuropeas antiguas se parecen mucho entre sí. Este parecido va disminuyendo posteriormente. Si el hetita, con ser antiguo, no se parece en esa medida a las otras lenguas, es porque no se trata de una lengua indoeuropea, sino de una rama hermana del indoeuropeo.

2) E1 hetita conserva una serie de arcaísmos de la época indo-hetita que el indoeuropeo ha perdido. A los ya mencionados con anterioridad añade ahora otros: abundancia de heteróclitos en $r / n$ : este sufijo sigue siendo productivo en hetita, mientras que en indoeuropeo se fosiliza.

3) Arcaísmos del hetita conservados, allí donde el indoeuropeo ha innovado, que para Sturtevant son los de mayor fuerza probativa: a los mencionados en trabajos anteriores añade aquí únicamente ciertos hechos de vocabulario. No menciona en cambio el problema relativo al género femenino.

En la segunda de sus obras mencionadas más arriba, la gramática hetita, presenta un esquema que puede adoptarse como compendio de su postura. La lengua común recibe el nombre de Proto-Indo-Hetita,

1 The indo-hittite hypothesis", Language 38, 1962, p. Iro ss.

- The indo-hittite laryngeals, Baltimore, 1942; $A$ comparative grammar of the Hittite Language, New Haven-London, 1964 (2.* ed., 1.* ed. 1933). 
y se aceptan nuevos arcaísmos hetitas en relación con el indoeuropeo, muy especialmente la ausencia de género femenino. En otros aspectos se sigue, en cambio, defendiendo el carácter innovador del hetita, como en lo referente al tema de perfecto, que el hetita habría perdido.

Si tenemos que emitir un juicio sobre el conjunto de las ideas de Sturtevant, diremos que su esfuerzo se encamina hacia la conservación con el mínimo de retoques posibles del indoeuropeo tradicionalmente reconstruido con anterioridad al desciframiento del hetita. El mismo proclama ${ }^{1}$ tener una visión neogramática de la lingüística indoeuropea y ser alumno fiel de maestros neogramáticos. No és de extrañar, pues, que insista y se esfuerce en mantener inalterada la lección aprendida.

Ahora bien, se da cuenta desde el primer mómento de que efectivamente los datos del hetita no concuerdan bien con la lección de sus maestros, de que difícilmente puede hacerse derivar el hetita del indoeuropeo neogramático, de que resultaría inverosímil suponer que todo en el hetita es innovación. Recurre entonces a un parentesco más lejano: se trataria no de lenguas "hermanas", sino de lenguas "primas" (son palabras textuales). E1 indoeuropeo tradicional sigue siendo válido, su reconstrucción correcta. Pero el hetita no procedería de ese indoeuropeo. A partir de ahora pueden admitirse todo tipo de arcaísmos para el hetita, sin miedo a poner en tela de juicio las conclusiones de la reconstrucción tradicional.

Sin embargo, Sturtevant no aprovecha hasta sus últimas consecuencias la libertad de movimientos que le otorga su hipótesis, quedándose en una posición intermedia poco satisfactoria. En efecto, como ya hemos visto, sólo admite ciertos arcaísmos - muy limitados en número- en la morfologia hetita, planteando en cambio una amplia gama de innovaciones en puntos de la flexión nominal y sobre todo verbal, que resultan poco verosímiles. En el fondo, el indo-hetita de Sturtevant no pasa de ser algo muy próximo al indoeuropeo de Brugmann, con ligeras modificaciones de detalles de menor cuantía.

Algunos de los lingüistas que aceptaron la idea de la temprana separación del hetita han sido más consecuentes con el punto de partida y han avanzado más en esa dirección, como luego veremos.

Lo que nos parece más duradero, al cabo de los años, del pensamiento de Sturtevant es la idea de una temprana separación del hetita, que, como vimos en su momento, no procede en realidad de él en última instancia. Por su parte, la formulación que dio a esa idea no resulta

1 Hittite and areal linguisticss, Language 23, 1947, pp. 377-385. 
afortunada al desenvolverse en el estrecho esquema del árbol genealógico. No cabe duda, sin embargo, de que su obra supuso en su momento un considerable avance, y que otros lingüistas coetáneos o posteriores adoptaron actitudes mucho más rígidas e intolerables para con el hetita. $\mathrm{Y}$ tuvo, desde luego, la virtud de dejar flotando en el ambiente la idea general de una temprana separación del hetita, aceptada después por muchos indogermanistas, algunos de los cuales prescindirán en cambio de la rigurosa formulación de Sturtevant.

Algunos lingüistas han aceptado literalmente la tesis del indo-hetita, en la formulación misma que le diera Sturtevant. De entre ellos, unos se limitan a tal aceptación, mientras que otros utilizan más a fondo las posibilidades que esta le ofrece ampliando el inventario de posibles arcaísmos de la rama anatolia.

Goetze ${ }^{1}$ considera decisivo el argumento de la conexión de los demostrativos indoeuropeos con los unidores de frases hetitas. Ên el mismo sentido se pronuncia A. Hahn ${ }^{2}$. Mansion ${ }^{3}$ admite igualmente la existencia en hetita de rasgos "très archaiques tel qu'aucune langue connue jusqu'à présent ne permettait reconnaitre. L'hypotèse d'un pre-indoeuropéen serait donc justifiée...».

Entre los lingüistas que aceptan la tesis del indo-hetita, ampliando el inventario de arcaísmos anatolios, podemos mencionar a Milewski, quien en un trabajo que no me ha sido accesible y sólo conozco de referencias acepta que categorfas como el acusativo de plural en - $n s$, el genitivo de plural en $-\varnothing m$ o el locativo de plural en $-s$ - no se habrian desarrollado aún en indo-hetita, siendo innovaciones puramente indoeuropeas posteriores a la separación del anatolio.

Kern-Schwartz ${ }^{4}$ opinan que la conjugación sobre varios temas que expresan nociones temporales de algunas lenguas indoeuropeas históricas fue precedida por otra más antigua que expresó sólo valores aspec-

\footnotetext{
1 "Hittite and indoeuropean languages", JAOS 65, p. $5 \mathrm{I}$ ss.

a Proceedings of the 8th International Congress of Linguists, Oslo, 1958, p. 248 .

3 J. Mansion, "A propos de la declinaison du hittite", M.langes linguistiques offerts a $H$. Pedersen, Copenhague, 1937, pp. $4^{80-487}$.

- "Multiple stem conjugation: an indo-hittite isogloss?", Language 22, 1946, pp. $57-68$.
} 
tuales. Pero advierten que los elementos formales que caracterizan al tema de presente frente al de aoristo en las demás lenguas indoeuropeas no están todavía en hetita dotados de valor especia 1 a $1 \mathrm{~g}$ uno. A veces mediante sufijos distintos se crean en hetita verbos distintos, totalmente sinónimos.

Consideran estos autores que en indo-hetita habría existido una verdadera conjugación aspectual de la que no habrían quedado sino fragmentos en las lenguas históricas, sin que se dé coincidencia ni en las formaciones conservadas por las distintas lenguas ni en el valor que presentan esas formaciones.

En lo que se refiere a las dos conjugaciones hetitas (en - $m i$ y en $-h i$ ), no creen los autores que demuestren la existencia en indo-hetita de dos flexiones distintas por su forma y su significación, sino todo lo más deben entenderse como ciertas posibilidades existentes de alternativa en el uso de las desinencias.

Tanto el aoristo como el perfecto de las lenguas indoeuropeas serían desarrollos posteriores a la separación del anatolio, mediante un procedimiento que los autores describen, y que en realidad resulta bastante verosímil. El hetita conservaría en este aspecto una situación más arcaica que cualquier lengua indoeuropea. La posibilidad contraria -carácter innovador del hetita - queda descartada por Kern-Schwartz como exenta de toda probabilidad ${ }^{1}$.

Diversos lingüistas, con un pensamiento total o parcialmente independiente de la hipótesis de Sturtevant, han ido poco a poco reconociendo otros arcaísmos del hetita.

Meillet ${ }^{2}$, haciendo especial hincapié en el problema del género femenino, su origen y desarrollo, admite la existencia de diversos arcaísmos en hetita, sobre todo el que acabamos de mencionar: el género femenino no ha sido eliminado por el hetita, sino que es una innovación del resto del indoeuropeo, de la que no habrían participado las lenguas anatolias por una parte, ni el armenio por otra.

1 En la misma línea de pensamiento W. Cowgill, More evidence for indohittite: the tense-aspect Systems", Proceedings of the IIth International Congress of Linguists, 1974, pp. 557-570.

- Essai de chronologie des langues indo-européennes", $B S L$ 32, 1931, pp. I-28. 
Para Burrow ${ }^{1}$ serla un arcaísmo del hetita, al igual que para KernSchwartz, su conjugación sobre un solo tema verbal. Lejos de haber dispuesto el hetita en fecha prehistórica de un sistema verbal similar al del indio o al del griego, serían estas lenguas las que habrian hecho derivar sus respectivos temas verbales de una situación similar a la del hetita de los textos históricos.

"The absence of distinction of meaning in all these types of formation between the present and aorist stem, in contradistinction to the clear distinction between the two types of preterite, points to the conclusion that the aorist developed as a special grammatical category. It appears that originally Indo-European distinguished in the indicative simply between present and preterite, the forms of wich could be made from a wide variety of stems. This $s$ ate of affairs is continued in Hittite, wich shows no sign of ever having had a tense corresponding to the aorist of other languages."

Burrow continúa postulando que el siguiente estadio de desarrollo es la creación de dos grupos de formas de pretérito, el uno con su correspondiente presente (imperfecto), y el otro desligado de todo presente (aoristo), y contando con un sentido especial: ésa es la situación atestiguada en indo-iranio. En griego, la distinción entre el tema de presente y el de aoristo es llevada más adelante y aplicada también a los modos, participios e infinitivo, derivados ya de ambos temas. En consecuencia, el pretérito del presente habría llegado por ese camino a adquirir un valor «imperfectivo" del que carecerfa todavía en het ita, y que también está ausente en las correspondientes formaciones del sánscrito.

Hamp ${ }^{2}$ aporta nuevos datos que abonan, a su entender, el carácter arcaico del hetita, esta vez procedentes de la fonética. Las labiovelares serían en las lenguas indoeuropeas verdaderos fonemas unitarios, y no grupos de fonemas. Sin embargo, en hetita la situación es distinta, equivaliendo a velar $+-w$ - La situación del anatolio a este respecto confirmaría lo que ya se sospechaba de antiguo: la independencia fonológica de las labiovelares se habría desarrollado en indoeuropeo tras la separación de la rama anatolia, resultando ser una innovación de capital importancia que afecta a todas las lenguas indoeuropeas con exclusión del hetita.

1 The Sanskrit Language, London, 1955, p. 296 ss.

2 Les labiovelaires en indo-européen et en anatolien, BSL 50, 1954, pp. 4446. 
Benveniste postula un nuevo arcaísmo, también fonético, para el hetita. Constata ${ }^{1}$ que la /s/ indoeuropea aparece testimoniada en hetita unas veces como /s/ y otras como /ts/ sin que se puedan establecer reglas o contextos que permitan suponer la escisión del fonema /s/ indoeuropeo en dos fonemas hetitas. De ello deduce Benveniste que debajo del fonema indoeuropeo /s/ se esconderian dos fonemas distintos, cuya oposicion se ha mantenido como arcaísmo en hetita, mientras que ambos se han llegado a fundir en las demás lenguas indoeuropeas: "Le hittite se révèle ainsi une fois encore comme le témoin d'une phase plus ancienne du phonétisme commun."

Más explícita es aún la opinión de Ivanov, quien defiende el carácter arcaico del hetita en diversos puntos de su morfología ${ }^{2}$. Los datos del hetita, para Ivanov, obligan a replantearse completamente la historia de la familia lingüística indoeuropea. En efecto, estos datos demuestran el desarrollo de las formas casuales de dual y plural, el origen de los relativos (jo-, $k^{*} e / 0-$ ) como elementos unidores de frase, etc., todo ello unido a otros rasgos arcaicos más frecuentemente mencionados por otros lingüistas. Para Ivanov, el hetita no sería el único superviviente de un estadio de lengua más arcaico que el indoeuropeo tradicional, sino que a su testimonio hay que unir el del tocario.

Las ideas, sobre el sistema verbal sugeridas : primero por KernSchwartz y luego por Burrow, con diferencias de detalle, van a ser recogidas por diversos lingüistas, adquiriendo en manos de cada uno ciertos matices personales, pero conservando el denominador común de considerar un arcaísmo del hetita el no contar con una flexión verbal montada sobre varios temas, portadores de valores aspectuales o temporales. Así entre otros Elizarenkova ${ }^{3}$.

Adrados, en un primer avance 4 , lanza en I96r una relación de hechos que él considera innovaciones producidas en el resto del indeuropeo con posterioridad a la separación del hetita, y que en consecuencia deben interpretarse como otros tantos arcaismos de la rama anatolia.

Los principales arcaismos que menciona son los siguientes:

1 Ettudes hittites et indo-européennes», BSL 50, 1954, p. 29 ss.

2 T. Ivanov, Proceedings of the 8th International Congress of Linguists, Oslo, I958, p. 250.

"On the problem of the development of tenses in old indo-aryan*, Paper... $25^{\text {th }}$ Congress of Orientalistic, Mosciu, 1960, p. 2 ss.

- F. R. Adrados, "Hethitisch und Indogermanisch" II Fachtagung für indogermanische und allgemeine Sprachwissenschaft (196I), Innsbruck, 1962, pp. 145-51. 
I. Reflejo de un estadio previo a la isoglosa centum/satəm.

2. Mantenimiento de las laringales consonánticas.

3. Carencia de la oposición masculino/femenino.

4. Situación de la flexión heteróclita.

5. Falta de aoristo en general y de aoristo sigmático en particular.

6. Ausencia de Subjuntivo.

7. La conjugación en - hi del hetita corresponde al perfecto del resto del indoeuropeo. Pero sería el hetita el que mantiene la situación antigua en que estos temas en laringal son un tema de presente más, a partir del cual el resto del indoeuropeo llegaría a desarrollar el tema de perfecto.

8. Abundancia de flexiones semitemáticas, que en el resto del indoeuropeo tendería a perderse.

9. Diversas particularidades en las desinencias verbales.

Esste trabajo al que acabamos de aludir no es en realidad sino un ad-lanto programático sobre la obra de conjunto que a prop6sito de la reconstrucción del verbo indoeuropeo estaba preparando. Esa obra ${ }^{1}$ es el trabajo más sistemático que se ha realizado por reconstruir el sistema verbal indoeuropeo manejando ya en profundidad los datos del hetita, en donde se razonan y justifican los diferentes arcaismos del sistema verbal hetita que ya se postulaban en el anterior trabajo mencionado. Frente al destronamiento del hetita que postulaba Kuryłowicz $^{2}$ no duda Adrados en afirmar que esta lengua debe convertirse en el centro mismo de la lingüística indoeuropea. Lo más asomibroso, para Adrados, es que el hetita presente de hecho la misnia situación lingüística a la que se llega por un análisis interno del sistema brugmanniano.

En el mismo año que la primera de estas publicaciones de Adrados aparece un trabajo de Gonda ${ }^{3}$, en el que el autor opina que la oposición presente/aoristo surge al crearse la serie primaria de desinencias en oposición a la secundaria preexistente: los temas en que se produjera la introducción de la nueva serie (primarias) serfan los presentes de

1 F. R. Adrados, Evolución y estructura del verbo indoeuropeo. Madrid, 1963 (2. ${ }^{\text {a }}$ ed. 1974).

2 J. Kurylowicz, "Le hittite", Proceedings of the 8th International Congress of Linguists, Oslo, 1958, pp. 216-243.

s The aspectual function of the Rigvedic present and aorist (Disputationes reheno-trajectinae, v. VII!, 1962. 
época histórica; los que continuaran con una sola serie (la secundaria) serían los aoristos de época histórica ${ }^{1}$.

De ello deduce Gonda que koriginariamente" no habría existido una distinción sistemática entre presente y aoristo, e $\mathrm{s} t \mathrm{ado} \mathrm{de} \cos \mathrm{s}$ que hay que pensar que continúa en hetita, que no presenta huellas de haber contadonunca con una categoria correspondiente al aoristo que se presenta en diversas 1 enguas indoeuropeas. $\mathrm{Y}$ lo que es más - continúa Gonda- en védico existen también huellas de esta situación: una forma del tipo sramat de tema no caracterizado puede ser interpretada como de presente o como de aoristo. No obstante, la situación del indo-iranio supone un avance, según Gonda, sobre la del hetita, y a su vez la del griego sobre la del indo-iranio ${ }^{2}$.

$\mathrm{N}$. van $\mathrm{Brock}^{3}$ emprende un detallado análisis de las formas verbales reduplicadas del hetita. Su estudio resulta muy instructivo en diversos aspectos que no es este el lugar de consignar. Nos limitaremos a decir que entre los diferentes tipos de reduplicación no encuentra la autora una verdadera diferencia funcional. El panorama en conjunto es, una vez más, muy distinto del que presentan las demás lenguas indoeuropeas. Se observan por otra parte múltiples indicios de un proceso de morfologización de las formas reduplicadas que está, sin embargo, muy lejos de haber llegado a su culminación.

*Pero de todo esto no se deduce necesariamente - dice van Brockque la reduplicación hetita no sea heredada y que no tenga nada en común con las formas reduplicadas de las otras lenguas. Pensamos -continúa diciendo la autora-que tenemos entre manos u $\mathrm{n}$ f e $\mathrm{n}$ ó $\mathrm{m}$ e $\mathrm{n}$ o único del que el hetita refleja únicamente un estadio más arcaico, menos evolucionado; y que en e 1 origen de 1 os hechos de las otras lenguas hay un estadio indoeuropeo bastante parecido a 1 que e1 hetita nos deja entrever."

Más adelante continúa diciendo: "Podemos afirmar que la rama anatolia se separó de la lengua madre en una época en que ésta poseía una serie indiferenciada de temas verbales reduplicados que implicaban por su misma formación un valor iterativo. Las formaciones anatolias deben representar bastante exactamente el estadio indoeuropeo de la

\footnotetext{
1 J. Gonda, op. cit., p. 270.

2 J. Gonda, $o p$. cit., pp. 270-271, cf. p. 263.

s Les thèmes verbaux à redoublement du hittite et le verbe indo-européens, Revue Hittite et Asianique 22, 1964, pp. 119-158.
} 
época de la separación, ya que se puede partir de la situación del hetita para explicar los desarrollos ulteriores en las demás lenguas. Un período de comunidad un poco más prolongado ha permitido a esas otras lenguas participar en una tentativa de diferenciación morfológica y de fijación normal de la reduplicación, proceso aún inacabado en la época de la separación definitiva, y la fijación final se ha llevado a cabo (o no se ha llevado a cabo) independientemente en las diferentes lenguas.n

A continuación critica van Brock a los lingüistas, especialmente Kronasser, que pretenden que el hetita haya perdido todo aquello que desde el punto de vista del indoeuropeo clásico se echa en falta en su morfologia, diciendo: "Para perder algo es preciso primero haberlo tenido. Es un hecho que el sistema verbal hetita resulta pobre en comparación con el verbo indoeuropeo de Brugmann; pero nada lleva a pensar que haya perdido todo lo que no posee...". "Es muy poco probable que el hetita haya arrojado por la borda un perfecto indoeuropeo recién creado para fabricarse inmediatamente otro (perifrástico). De hecho el hetita nunca llegó a tener un perfecto reduplicado, ni siquiera un perfecto... Resulta más que dudoso que el indoeuropeo poseyera un perfecto en la época de la separación del hetita."

En resumen, nos encontramos en este trabajo de $\mathrm{N}$. van Brock con una opinión que considera arcaico al hetita en relación con la categoría del perfecto en particular, y de la reduplicación como recurso morfológico del sistema verbal en general, en contra de Sturtevant, que opinaba que el hetita había perdido el perfecto indoeuropeo. Igualmente Gonda ${ }^{1}$ se inclina a pensar que la equivalencia funcional de las flexiones verbales en $-m i$ y $-h i$ es fruto de una innovación del hetita, y que en consecuencia el hetita tuvo y perdió el perfecto heredado del indoeuropeo.

Como puede verse, los arcaísmos del hetita son detectados, generalmente, en la fonética y en el sistema verbal ${ }^{2}$. Son poco numerosos, en cambio, los trabajos que hacen referencia a posibles arcaísmos del grupo anatolio dentro del campo nominal, con algunas excepciones,

1 Gonda, op. cit., p. 263.

2 Veanse trabajos más recientes sobre la reconstrucción del sistema verbal indoeuropeo de acuerdo con los datos del hetita, que sin embargo no aporta nada sustancialmente nuevo a lo hasta aquí mencionado, en Neu, "Zur Rekonstruktion des indogermanischen Verbalsystems", Studies in Greek, Italic and Indo-European Linguistics, offered to L. R. Palmer, Innsbruck, 1976, pp. 239254 cuyos datos no he podido tener en cuenta en el cuerpo de este trabajo, por haber llegado a mis manos cuando estaba ya ultimada la redacción definitiva. 
como los ya mencionados Meillet, Ivanov y Milewski, y los que ahora mencionaremos.

Por mi parte ${ }^{1}$, he tratado de aislar las posibles isoglosas que en el desarrollo de la flexión nominal de la familia lingüística indoeuropea no afectan al hetita, para ensayar luego un panorama del posible proceso de creación de las categorías flexivas nominales. Entre ellas pueden citarse la ausencia de género femenino que ya señalara Meillet ${ }^{2}$, a 10 que hay que añadir verosímilmente la precaria situación de la oposición animado/inanimado; ausencia de dual; escaso desarrollo del plural; identidad nominativo-genitivo en el singular del tipo temático, etc. Ideas similares defiende Adrados en un reciente trabajo (véase más abajo), aunque con diferencias en determinados puntos concretos de la reconstrucción.

Más recientemente, Carruba ${ }^{3}$ admite diversos arcaísmos en hetita: 1a falta de femenino, la incipiente situación de la oposición animado/ inanimado, la ausencia de gradación en el adjetivo, la falta de aoristos especialmente sigmáticos, etc. Opina, en cambio, que el hetita contó con un perfecto de estado, perdido como tal, pero testimoniado en la conjugación en - $h i$. En conjunto cree que el grupo anatolio puede considerarse como altamente arcaico en relación con el resto de las lenguas de la familia indoeuropea.

IV

Una posición en cierto modo intermedia entre los lingüistas que consideran arcaica la situación lingüística del hetita en uno u otro punto, y la de los que consideran el grupo anatolio totalmente innovador, que se ha alejado por una abundante serie de innovaciones del esquema lingǘstico del indoeuropeo neogramático, es la que mantiene Petersen.

Admite, por una parte, este lingüista una temprana separación del hetita, mostrándose en cambio contrario a toda interpretación de su situación lingüística como arcaísmo. Opina ${ }^{4}$ que el hetita ha perdido

1 F. Villar, Origen de la flexión nominal indoeuropea, Madrid, C. S. I. C., 1974 .

2 A. Meillet, "Essai de chronologie des langues indo-européennes", $B S L 32$, I93I, pp. I-28.

"Anatolico e indoeuropeon, Scritti in Onore G. Bonfante, Brescia, 1976, vol. I, p. I2I ss.

- Personal endings of the hittite verb*, $A J P h$ 53, 1932, p. 103 ss. 
o confundido el aoristo, el perfecto, el dual, etc. Las desinencias verbales hetitas pueden explicarse desde las del indoeuropeo clásico. Unicamente quizás el futuro en -sịo- y el aumento podrían ser desarrollos posteriores a la separación del hetita. I os restos del aoristo en $-s$ - se encuentran en ciertas desinencias que contienen ese elemento ${ }^{1}$. El perfecto indoeuropeo se habría escindido, dando por una parte los presentes en -hi (cf. los perfecto-presentes del germánico, etc.) y fundiéndose por otra con el imperfecto y el aoristo para el pasado de la conjugación en -mi. El pasado de la conjugación en - hi sería secundario, como los pasados compuestos de los perfecto-presentes germánicos. En un artículo anterior ${ }^{2}$ admite el carácter reciente del plural en la flexión nominal para la mayoría de las formas casuales, no sólo para el hetita, sino para el indoeuropeo en general. En este sentido, a diferencia de lo que opinan otros lingüistas, para Pedersen las lagunas en la expresión de los casos en plural que presenta el hetita no serian efecto de una pérdida, sino que nunca habrían llegado a desarrollarse.

Simultánea a la obra de los lingüistas que admiten el carácter arcaico del hetita en todo o en parte, un grupo igualmente numeroso de estudiosos defiende por el contrario de una manera rígida el carácter innovador del grupo anatolio. Entre las figuras más representativas de esta tendencia podemos mencionar a Pedersen ${ }^{3}$, Friedrich ${ }^{4}, K_{\text {Konnaser }}{ }^{6}$, Kuryłowicz ${ }^{6}$, Kammenhuber ${ }^{7}$, etc.

1 Véanse ideas similares en Kammenhuber, "Zur Stellung des HettitischLuvischen innerhalb der indogermanischen Gemeinsprachey, $K L 77$, 196r, p. 31 ss., *Hethitisch, Palaisch, Luwisch und Hieroglyphenluwisch", Altkleinasiatische Sprachen, Leiden, 1969, pp. I19-354; Eichner, "Die Vorgeschichte des hethitischen Verbalsystemsw, Flexion und Wortbildung, 1975, p. $7 \mathrm{I}$ ss.; etc.

2 "Hittite and indo-european plural declension", $A J P h$ 5I, 1930, pp. $25 \mathrm{I}-72$.

- H. Pedersen, Hittitisch und die audere indo-europäischen Sprachen, Copenhague, 1938; Lykisch und Hittitisch, Copenhague, 1945.

- J. Friedrich, "Die hethitische Sprache», $Z D M G, N F$ 1, 1922, p. 147 ss.; Hethitisch und Kleinasiatische Sprachen, Berlin-Leipzig, 1931; Staatsverträge des Hatti-Reiches I, 1926, II, 1930. Leipzig; Hethitisches Elementarbuch, Heidelberg, 1960 (1.* ed. 1940-46); Entzifferung verschollener Schriften und Sprachen, Berlin, 1954 .

- H., Kronasser, Vergleichende Laut- und Formenlehre des Hethitischen, Heidelberg, 1946; Etymologie der hethitischen Sprache, Wiesbaden, r962-66.

- J. Kuryłowic.:, "Le hittite», Proceedings of the 8th International Congress of Linguists, Oslo, 1958, pp. 216-251; "L,es desinences moyennes de l'indo-européen et du hittites, $B S L$ 33, 1932, p. I ss.; The inflexional cathegories of indo-enropean, Heidelberg, 1964 .

7 A. Kammenhuber, ¿Zur hethitisch-luwischen Sprachgruppe», $K Z$ 76, 1960, 
Carece de interés entrar en un análisis en detalle de las doctrinas de estos lingüistas, dados los propósitos de estas páginas. En términos generales, para ellos el hetita tendría un interés casi nulo en la reconstrucción de la historia de la familia lingüística indoeuropea ya que su testimonio no es de un estadio lingüístico más arcaico que el de las otras lenguas anteriormente conocidas, sino por el contrario presenta una situación mucho más evolucionada y alterada. Nada aporta el hetita, o casi nada, a la reconstrucción del indoeuropeo. Por el contrario, como suponen que el hetita partió de una situación lingüística similar a la del indoeuropeo brugmanniano, es ese indoeuropeo el que puede ayudar a penetrar en la prehistoria de las lenguas anatolias. Ese es el principio general del que parte esta corriente opinión.

El problema, entonces, consiste en ir viendo dónde han ido a parar las distintas categorias gramaticales del complejo sistema brugmanniano, así como qué ha quedado de sus significantes, averiguar la proporción de influencias externas, etc.

Esta actitud de considerar innovador al hetita fue inaugurada por Pedersen ${ }^{1}$ a los diecinueve años del desciframiento de los textos hetitas por parte de Hrozny. Antes de ese trabajo de Pedersen había predominado la opinión contraria en forma casi monopolística: el hetita es en conjunto más arcaico que el resto de las lenguas indoeuropeas. Esta actitud, con anterioridad al mencionado trabajo de Pedersen, puede estar simbolizada por el trabajo de Marstrander ${ }^{2}$, y que abonada por la identificación hecha por Kuryłowicz ${ }^{3}$ de la $h$ del hetita con las laringales postuladas por la teoría para el indoeuropeo, tuvo su punto culminante en los diferentes trabajos de Sturtevant que ya han quedado analizados.

La corriente opinión que ve en el hetita una lengua fuertemente innovadora, tras la propuesta de Pedersen se ve en seguida reforzada por la adhesión de Sommer ${ }^{4}$, y alcanza a su vez su punto culminante en la obra de Kronasser ${ }^{5}$, así como en la intervención de Kuryłowicz

\footnotetext{
p. I ss.; "Zur Stellung des Hethitisch-Luwischen innerhalb der indogermanischen Gemeinsprache", $K Z$ 77, I96r, p. 31 ss.; "Hethitisch, Palaisch, Luwisch und Hyeroglyphenluwisch», Altkleinasiatische Sprachen, Leiden, 1969, pp. Ir9-354.

1 Hittitisch und die andere indo-europäischen Sprachen, Copenhague, 1938.

- Charactere indo-européen de la langue hittite, Christiania, 1919.

- J. Kuryłowicz, sL'indo-européen et $h$ hittite», Symbolae Grammaticae in ho-

norem I. Rozwadowski, Cracovia, 1927, vol. I, pp. 95-ror.

- Hethiter und Hethitisch, Stuttgart, 1947, p. 45.

- C. p. $18_{5}$, n. 5 .
} 
en el Octavo Congreso Internacional de Lingüistas ${ }^{1}$. A partir de ahí puede considerarse que la opinión de los lingüistas está dividida a este respecto, $\sin$ que la balanza se haya inclinado definitivamente hacia una de las dos posibilidades. Recientemente ha habido pronunciamientos en ambos sentidos, como el de Carruba ${ }^{2}$ en favor del carácter arcaico, o la de Eichner ${ }^{3}$, precisamente en sentido contrario. Para W. Eichner el sistema verbal hetita no es reflejo de una etapa más arcaica de la familia lingǘstica indoeuropea, sino el fruto de la reorganización de los elementos comunes heredados. Aún quedarían reflejos en anatolio de categorías como el dual, el subjuntivo, el aumento, el perfecto reduplicado y las características del aoristo. En cambio, han sido totalmente eliminados el subjuntivo en o/e, el optativo de tipo atemático, la oposición aspectual presente/aoristo, etc.

Este es el dilema crucial de la indogermanística en el momento presente. De aceptar una u otra de ambas posibilidades depende que se trace una historia de la familia lingǘstica indoeuropea de muy diferente signo. Si el hetita es una lengua más arcaica, en diferentes puntos de su morfología y de su fonética, que las demás lenguas indoeuropeas que sirvieron para la reconstrucción de estilo neogramático, evidentemente su testimonio nos ayudará a alcanzar estadios más antiguos, niveles sincrónicos más arcaicos. En cambio, si el hetita es una lengua radicalmente innovadora, hay que seguir manteniendo el esquema brugmanniano a grandes rasgos, y las aportaciones de los indoeuropeístas deberán centrarse en perfeccionar ésta o aquella cuestión de detalle. Aunque, como deciamos más arriba, la situación del hetita es curiosamente la misma que se podía deducir mediante un análisis interno del sistema brugmanniano para un estrato profundo de la evolución del grupo indoeuropeo.

La importancia y trascendencia de la actitud que se adopte a este respecto debe mover a los indoeuropeístas a tomar posición sin dejarse llevar de ningún tipo de prejuicios y sin miedo a la tarea de una nueva

\footnotetext{
1 J. Kuryłowic\%, "Le hittite", Proceedings of the 8th International Congress of Linguists, Oslo, 1958, pp. 216-243.

2 Véase lo dicho en p. 184 .

- "Die Vorgeschichte des hettitischen Verbalsystems", Flexion und Woribildung, 1975, p. 71 ss.
} 
reconstrucción como hemos visto que postulaba Ivanov en el octavo congreso de lingüistas. Un intento en este sentido, para el sistema verbal, es el realizado, como ya queda dicho, por Adrados ${ }^{1}$. Otro tanto he querido hacer yo para el sistema nominal ${ }^{2}$. Y en conjunto, para toda la morfología, así como para la fonética, Adrados ha ensayado uua sistematización en su más reciente libro al que también hemos ya aludido ${ }^{3}$.

F. VILLAR

1 F. R. Adrados, Evolución y Fstructura del verbo indoeuropeo. Madrid, r963.

2 F. Viliar, Origen de la Flexión Nominai Indoetsropea. Madrid, r974.

- F. R. Adrados, Lingüistica Indoeuropea. Madrid, 1975. 